\title{
In vivo efficacy of the histone deacetylase inhibitor suberoylanilide hydroxamic acid in combination with radiotherapy in a malignant rhabdoid tumor mouse model
}

Markus Thiemann ${ }^{1 \dagger}$, Susanne Oertel ${ }^{1 *}$, Volker Ehemann ${ }^{2}$, Wilko Weichert ${ }^{2}$, Albrecht Stenzinger $^{2}$, Marc Bischof ${ }^{1}$, Klaus-J Weber', Ramon Lopez Perez ${ }^{3}$, Uwe Haberkorn ${ }^{4}$, Andreas E Kulozik ${ }^{5}$, Jürgen Debus ${ }^{1}$, Peter E Huber ${ }^{1,3}$ and Claudia Battmann ${ }^{5}$

\begin{abstract}
Purpose: Histone deacetylase inhibitors are promising new substances in cancer therapy and have also been shown to sensitize different tumor cells to irradiation (XRT). We explored the effect as well as the radiosensitizing properties of suberoylanilide hydroxamic acid (SAHA) in vivo in a malignant rhabdoid tumor (MRT) mouse model.

Methods and material: Potential radiosensitization by SAHA was assessed in MRT xenografts by analysis of tumor growth delay, necrosis (HE), apoptosis (TUNEL), proliferation (ki-67) and $\gamma \mathrm{H} 2 \mathrm{AX}$ expression as well as dynamic ${ }^{18} \mathrm{~F}$ Fluorodeoxyglucose Positron Emission Tomography $\left({ }^{18} \mathrm{~F}\right.$-FDG -PET) after treatment with either SAHA alone, singledose (10 Gy) or fractionated XRT (3 × 3Gy) solely as well as in combination with SAHA compared to controls.

Results: SAHA only had no significant effect on tumor growth. Combination of SAHA for 8 days with single-dose XRT resulted in a higher number of complete remissions, but failed to prove a significant growth delay compared to XRT only. In contrast fractionated XRT plus SAHA for 3 weeks did induce significant tumor growth delay in MRTxenografts.

The histological examination showed a significant effect of XRT in tumor necrosis, expression of Ki-67, $\gamma \mathrm{H} 2 \mathrm{AX}$ and apoptosis. SAHA only had no significant effect in the histological examination. Comparison of xenografts treated with XRT and XRT plus SAHA revealed a significantly increased $\gamma \mathrm{H} 2 \mathrm{AX}$ expression and apoptosis induction in the mice tumors after combination treatment with single-dose as well as fractionated XRT. The combination of SAHA with XRT showed a tendency to increased necrosis and decrease of proliferation compared to XRT only, which, however, was not significant. The ${ }^{18}$ F-FDG-PET results showed no significant differences in the standard uptake value or glucose transport kinetics after either treatment.
\end{abstract}

Conclusion: SAHA did not have a significant effect alone, but proved to enhance the effect of XRT in our MRT in vivo model.

Keywords: Malignant rhabdoid tumor, In vivo, Radiotherapy, Histone deacetylase inhibition, Suberoylanilide hydroxamic acid

* Correspondence: susanne.oertel@med.uni-heidelberg.de

† Contributed equally

${ }^{1}$ Department of Radiation Oncology, University of Heidelberg, INF 600, 69120

Heidelberg, Germany

Full list of author information is available at the end of the article 


\section{Introduction}

The aim of the present study was to evaluate the in vivo efficacy of the HDACi SAHA in combination with XRT in MRT xenotransplants.

MRT and their central nervous system counterpart atypical teratoid/rhabdoid tumors are rare and highly malignant neoplasms that primarily occur in young children. Emerging evidence suggests an important role for radiotherapy to achieve long-term survival. Nevertheless even after toxic multimodal treatment including radiotherapy outcome is generally poor and new therapy options are urgently needed $[1,2]$. Therefore interest in adjuvant agents that selectively augment the response of MRT to radiation and thus increase the therapeutic ratio is high. Histone deacetylase inhibitiors (HDACi) are under investigation in anti-cancer treatment. They work by epigenetic regulation of gene expression, induce cell growth arrest, apoptosis as well as terminal differentiation. Clinical trials so far indicate moderate toxicity and favorable safety profiles. Even though in single-agent treatments only moderate improvements in outcome were observed [3], the combination of HDACi with other therapy modalities like radiotherapy has been reported to improve the therapeutic effect in many tumor entities in cell lines and xenograft tumor models [4-7]. We recently reported that the HDACi suberoylanilide hydroxamic acid (SAHA) radiosensitized pediatric sarcoma and rhabdoid tumor cell-lines in vitro through increase of apoptosis and regulation of the cell cycle as well as down-regulation of DNA-repair proteins [8]. Knipstein et al. reported that histone deacetylase inhibitors decrease proliferation and potentiate the effect of ionizing radiation in two further rhabdoid tumor cell lines [9].

Our data as well as the data of other groups showed that the radiosensitization through HDACi is associated with increased expression of $\gamma \mathrm{H} 2 \mathrm{AX}$ in treated tumor cell lines, supporting the theory that changes in XRTinduced DNA damage repair plays a role in the radiosensitization of tumor cells by HDACi [10]. $\gamma$-H2Ax expression was therefore investigated in the treatment xenograft specimen along with proliferation, apoptosis and necrosis as further possible underlying mechanisms.

Measurement of glucose metabolism with positron emission tomography (PET) has become an important imaging modality in the non-invasive evaluation and monitoring of malignant diseases. In sarcoma patients, ${ }^{18}$ F-Fluorodeoxyglucose Positron Emission Tomography $\left({ }^{18} \mathrm{~F}\right.$-FDG PET) has been shown to be useful in staging, therapy monitoring, and detection of relapse [11]. The use of PET/CT using F-FDG in rare tumors like MRT is unclear. Therefore, we were interested in the correlation of glucose metabolism with clinical and histopathological outcome in our xenograft mouse model and included ${ }^{18}$ F-FDG PET studies in the first part of our mouse experiments.

\section{Materials and methods \\ Cells and reagents}

The A-204 human MRT cell line (which is falsely claimed to be a rhabdomyosarcoma cell line) was obtained from the American Type Culture Collection (ATCC; Rockville, MD) and maintained in a complete culture medium (McCoy) supplemented with 10\% FCS. SAHA was obtained from Alexis Biochemicals (Lörrach, Germany), DMSO from Carl Roth Biochemicals (Karlsruhe, Germany). Ketamine $(0.4 \mathrm{mg} / 20 \mathrm{~g} \mathrm{BW})$ and xylazine (Bayer Germany) $(90 \mathrm{mg} / 20 \mathrm{~g} \mathrm{BW})$ were used to anesthetize mice during radiation treatments.

\section{Animal model}

Xenografts of human MRT cells were established by subcutaneous inoculation of $5 \times 10^{6}$ A-204 cells into the hind legs of 10 weeks old female $\mathrm{BALB} / \mathrm{cNu} / \mathrm{Nu}$ athymic mice (Charles River, Wilmington, Mass.). The mice were maintained under specific pathogen-free conditions, food and water were supplied ad libitum. Housing and all procedures involving the mice were performed according to the protocols approved by the local regional board.

Two trial cohorts were treated with slight differences in the treatment regimen.

In our first cohort mice were randomly assigned to the different treatment groups, when tumors reached $300 \mathrm{~mm}^{3}$ as PET-imaging prior to therapy was only possible in rather big tumors. In our second xenograft cohort we set aside PET-imaging and assigned the tumors to the different treatment arms when they reached $100 \mathrm{~mm}^{3}$.

Cohort I consisted of 12 mice per treatment group, Cohort II of 10 mice per treatment group.

The four treatment arms were as follows:

1) Vehicle (DMSO) control,

2) SAHA alone $(100 \mathrm{mg} / \mathrm{kg}$ for 8 days (first cohort of mice) or 21 days (second cohort of mice)

3) XRT alone (with $1 \times 10$ Gy in the first cohort and $3 \times 3$ Gy on 3 consecutive days in the second cohort) and Combination treatment of SAHA with XRT (correspondingly, Cohort 1 :

4) SAHA for 8 days $+1 \times 10$ Gy XRT, Cohort II: SAHA for 15 days ( 5 days/week for 3 weeks) $+3 \times 3$ Gy XRT).

Initially each group of the first cohort consisted of 13 mice. 1 mouse in the XRT group and 2 mice in the group with combination treatment died during anesthesia-related complications before XRT. In the second cohort each treatment group consisted of 10 mice. 
All mice were sedated with ketamine/xylazine at the days, when PET-imaging or XRT were performed (day $-1,0$ and day 8$)$.

SAHA $100 \mathrm{mg} / \mathrm{kg}$ was solubilized in DMSO (99.5\%), given intraperitoneally (i.p.), starting $24 \mathrm{~h}$ before XRT. The decision to start the SAHA treatment $24 \mathrm{~h}$ before XRT was based on our in vitro data that showed the highest sensitization of tumor cells after incubation for 24 hours [8]. On day 1 , mice tumors were irradiated with a single dose of 10 Gy (Cohort I) and $3 \times 3 \mathrm{~Gy}$ (Cohort II), respectively, directed at the tumor site. This dose corresponds to a tumor growth delay of 20 days which was determined in a preceding test. During the follow-up mice were weighed, and the tumor sizes were measured using a calliper twice a week. Tumor length (L) and width (W) were measured and tumor volume calculated as $\left(\mathrm{L} \times \mathrm{W}^{2} / 2\right)$, where $\mathrm{L}=$ longest diameter and $\mathrm{W}=$ shortest diameter.

Animals were euthanized by $\mathrm{CO}_{2}$ inhalation followed by cervical dislocation when tumors reached $3.5 \mathrm{~cm}^{3}$ or latest 65 days after treatment start.

\section{F-18 Fluorodeoxyglucose Positron Emission Tomography $\left({ }^{18} \mathrm{~F}-\mathrm{FDG}-\mathrm{PET}\right)$}

Dynamic ${ }^{18}$ F-FDG-PET measurements were performed in 5 mice per treatment group prior to treatment (day 0 for the untreated control group and XRT group, day -1 for the SAHA or SAHA plus XRT treated group) and at the end of treatment (day 8). The PET studies were performed in list mode for $60 \mathrm{~min}$ after intraveneous (i.v.) administration of $6 \mathrm{MBq}{ }^{18} \mathrm{~F}-\mathrm{FDG}$ (inhouse production, German Cancer Research Centre Heidelberg, Germany) using a matrix of $256 \times 256$ (pixel size $0.3882 \times 0.3882 \times 0.796 \mathrm{~mm}$ ). There after images were reconstructed at definite time periods after tracer administration $(2 \times 15 \mathrm{~s}, 8 \times 30 \mathrm{~s}, 5 \times 60$ s, $4 \times 120$ s, $2 \times 210$ s, $7 \times 300$ s). The images were reconstructed iteratively using the space alternating generalized expectation maximization method (SAGE, 16 subsets, 4 iterations) applying median root prior correction. Time-activity curves were created using volumes of interest (VOIs) over the hearts (input function) and the tumors. For the input function and the tissue response curve we used the maximal value of the VOI data. The following parameters were retrieved from the dynamic PET studies: standardized uptake value (SUV) at 50-60 minutes after tracer administration and the kinetic parameters k1, k2, k3, k4 and VB obtained form a pharmacokinetic analysis and the FDG influx calculated using the formula: influx $=(\mathrm{k} 1 * \mathrm{k} 3) /$ $(\mathrm{k} 2+\mathrm{k} 3)$. A dedicated micro PET system (Inveon, Siemens, Erlangen, Germany) was used for the animal studies. The evaluation of the PET-data was performed using the software package PMOD.

\section{Immunohistochemical analysis}

Histomorphological analysis was done in three tumor samples of each treatment group of Cohort I on day 8 (corresponding to the last day of SAHA treatment) and at the last day of observation (day 60 at the latest). In Cohort II histological samples were taken on day 21 (corresponding to the last day of SAHA application in this Cohort). Tumors excised from euthanized mice were formalin-fixed and paraffin-embedded. $1 \mu \mathrm{M}$ thick sections were cut and stained with hematoxylin-eosin (HE; Sigma-Aldrich, St Louis, USA). Subsequently, 3 $\mu \mathrm{M}$ thick sections were cut for immunohistochemistry. Staining for Ki-67 and Myo-D1 was performed as follows: First, tissue slides were deparaffinated with xylol and ethanol. To improve antigen retrival slides were cooked in $0.1 \mathrm{M}$ citrate. Primary antibodies (monoclonal mouse anti-MyoD1 clone 5.8 A (1:50, over night, $4{ }^{\circ} \mathrm{C}$; Dako, Hamburg, Germany), monoclonal mouse antihuman Ki-67 clone MIB-1 (1:200, overnight $4^{\circ} \mathrm{C}$; Dako, Hamburg, Germany)) were added. Then, a biotinylated anti-mouse secondary antibody was administered for 25 min. Endogenous peroxidase was blocked with $\mathrm{H}_{2} \mathrm{O}_{2}$ for 7 min. Slides were incubated with a streptavidin HRP construct (25 min,, Biolegend, San Diego, USA). Subsequently, slides were stained with 3-Amino-9-ethylcarbazole (AEC; Sigma-Aldrich, St Louis, USA) and counterstained with hemalaun (AppliChem, Darmstadt, Germany).

Apoptosis was quantified by the in situ apoptosis detection kit, ApopTAG ${ }^{\circledR}$ (S7100; Chemicon International (Millipore), Temecula, CA, USA) according to the manufacturer's instructions.

All tissue evaluation was done by two trained pathologists (WW and AS).

$H \& E$ stainings were evaluated with respect to tumor cell pleomorphism, number of mitotic cells and extent of necrosis, estimated visually by an experienced pathologist. Ki-67 and Myo-D1 staining were quantified by Spectrum $^{\mathrm{TM}}$ software (Aperio, Vista CA, USA).

TUNEL-positive cells were counted in ten high power fields of each case and a mean was calculated.

\section{Flow cytometry}

$\gamma \mathrm{H} 2 \mathrm{AX}$ expression was assessed in three xenografts per treatment group using flow cytometry. Tumor cells were separated in a solution of $2.1 \%$ citrate acid and $0.5 \%$ tween. Tumor cells of three mice per group were washed with PBS several times and then fixed with $3 \%$ paraformaldehyde (PFA, Sigma) for $10 \mathrm{~min}$ at $37^{\circ} \mathrm{C}$. Icecold methanol (90\%) was added and samples were kept on ice for another $30 \mathrm{~min}$. Afterwards, samples were washed three times in $0.5 \% \mathrm{PBS} / \mathrm{BSA}$, resuspended in $100 \mu \mathrm{l} 0.5 \% \mathrm{PBS} / \mathrm{BSA}$ and incubated for $10 \mathrm{~min}$ at RT. To stain the cells for $\gamma \mathrm{H} 2 \mathrm{AX}$, the antibody (anti- 
phospho-Histone H2AX (Ser 139), clone JBW301) from Millipore (Molsheim, Germany) was diluted 1:10 in 0.5\% PBS/BSA and $100 \mu \mathrm{l}$ solution was applied per sample. After $1 \mathrm{~h}$ incubation time at RT, cells were washed another three times in PBS/BSA. Cells were further stained with DAPI for $30 \mathrm{~min}$ on ice. The samples were analyzed directly on a "BD ${ }^{\mathrm{TM}} \mathrm{LSRII}$ "- flow cytometer from "Becton, Dickinson and Company (Franklin Lakes, New Jersey US)". The relative fluorescence intensity in the gated areas was detected using the multiparameter "BD FACSDiva ${ }^{\mathrm{TM}}$ " from "Becton, Dickinson and Company (Franklin Lakes, New Jersey US)" and analysed with the software "FlowJo7.6.5" by "Tree Star Inc". To assess the mean extent of DNA damage at a particular phase of the cell cycle, the mean values of $\gamma \mathrm{H} 2 \mathrm{AX}$ immunofluorescence (IF) were calculated separately for $\mathrm{G}_{0 / 1}, \mathrm{~S}$ and $\mathrm{G}_{2} \mathrm{M}$ cells by the computer-interactive "gating" analysis. Cells in $\mathrm{S}$ and $\mathrm{G}_{2} \mathrm{M}$ have a 1.5 respectively 2.0 higher $\gamma \mathrm{H} 2 \mathrm{AX}$ mean IF compared to cells in $\mathrm{G}_{0 / 1}$ because of the increase of DNA and histone content during the cell cycle. Therefore, the data has to be normalized for DNA (histone) content by dividing the mean $\gamma \mathrm{H} 2 \mathrm{AX}$ IF of $\mathrm{S}$ - and $\mathrm{G}_{2} \mathrm{M}$-phase cells by 1.5 and 2.0 , respectively. Finally, a low level of $\gamma \mathrm{H} 2 \mathrm{AX}$ IF is seen in the untreated cells which represent an "intrinsic" $\gamma \mathrm{H} 2 \mathrm{AX}$ phosphorylation. Therefore, the $\gamma \mathrm{H} 2 \mathrm{AX}$ IF level of the untreated controls has to be subtracted from the IF level of the treated cells in order to get the $\gamma \mathrm{H} 2 \mathrm{AX}$ IF level which is treatment-related.

\section{Data analysis}

Actual tumor growth delay was calculated with $\left(\mathrm{T}_{\mathrm{x}}{ }^{\prime}-\mathrm{T}_{\mathrm{x}}\right) /$ $T_{x}$ as the time taken for the irradiatied tumors ( $\left.T^{\prime}\right)$ and the control tumors $(\mathrm{T})$ to $\mathrm{x}$-fold multiply their volume (x).

The two-sided $t$-test was used to analyze the differences between the treatment groups. All data are presented as the mean $+/$ - standard deviation. $P$ values < 0.05 were considered statistically significant. Local Control (LC) was defined as the time from the initiation of treatment until the time a tumor had reached $\geq 1000$ $\mathrm{mm}^{3}$ in size. LC was estimated by Kaplan-Meier curves, and the differences in TTF between groups were assessed using log-rank test.

\section{Results}

It was previously shown that SAHA is able to enhance radiosensitivity in MRT cells in vitro [8,9]. To extend these findings, we now investigated the in vivo radiosensitizing potential of SAHA in a MRT xenograft mouse model.

During the first 5 days of SAHA treatment, the animals suffered from loss of appetite. Even though the mice recovered soon application of SAHA was therefore not continued consecutively, but discontinued during weekends in the second cohort and well tolerated. Mice were weighed twice a week, body weight remained tolerable (maximum weight loss was $8 \%$ and similar in all treatment groups.

Treatment with XRT respectively XRT plus SAHA delayed the tumor growth rate compared to treatment with SAHA alone or untreated controls. After a followup of $>40$ days our data showed a trend towards slower tumor progression in the combined treatment group compared to xenotransplants treated with XRT alone, but this was not statistically significant in Cohort I treated with single-dose XRT and SAHA for 8 days $(p=$ 0.3) (Figure 1a). However, we were able to prove statistical significance in the second cohort, treated with $3 \times$ 3 Gy fractionated XRT and SAHA for 3 weeks $(p<0.05)$ (Figure 2) We calculated local control (absences of local failure defined as tumor growth $>1000 \mathrm{~mm}^{3}$ ) according to the method of Kaplan and Meier in both cohorts. In Cohort I 50 days after start of treatment $20 \%$ of mice in the combination group compared to $0 \%$ of mice in the XRT had not experienced local failure (Figure 3). In cohort II, in which treatment started when tumors were much smaller, the same trend was intensified with a local control rate of $85 \%$ versus $25 \%$ after 60 days in favor of the combined treatment group. SAHA as a single-agent had no influence on local control in our

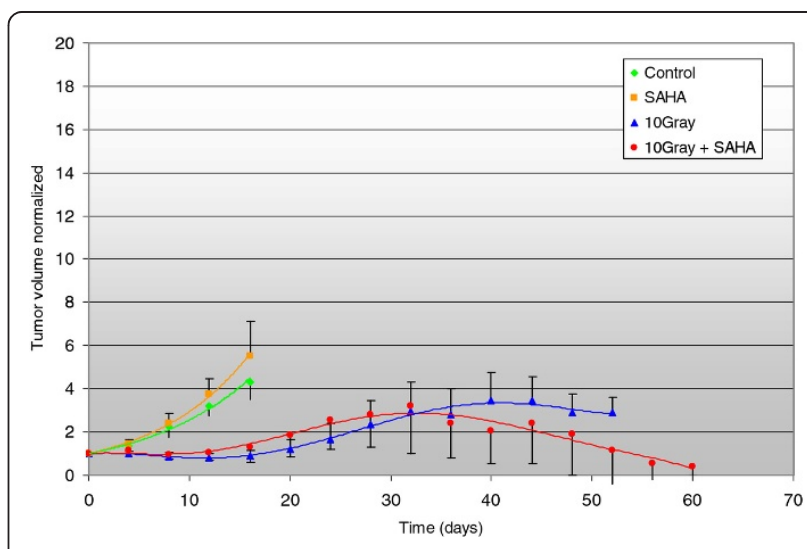

Figure 1 Tumor progression of malignant rhabdoid tumor xenografts (MRT) in Cohort I after treatment with ionizing radiation (XRT) or SAHA and XRT. The $y$-axis plots the multiplication of initial tumor size normalized to the initial size $=1$, $x$-axis plots the time after treatment initiation in days. Figure 1 represents Cohort I and Figure 2 Cohort II. SAHA $100 \mathrm{mg} / \mathrm{kg}$ was injected intraperitoneally once daily for 8 consecutive days in Cohort I and for 15 days within 3 weeks in Cohort II starting with day 0 . XRT with a single dose of $10 \mathrm{~Gy}$ (Cohort I) or with $3 \times 3 \mathrm{~Gy}$ on three consecutive days (Cohort II) was delivered day $1(-3)$. After a follow-up of $>40$ days, there was a trend towards slower progression in the combined treatment group compared to xenotransplants treated with XRT alone in Cohort I $(p=0.3)$, and a significant difference in Cohort II. $(p<0.05)$. 


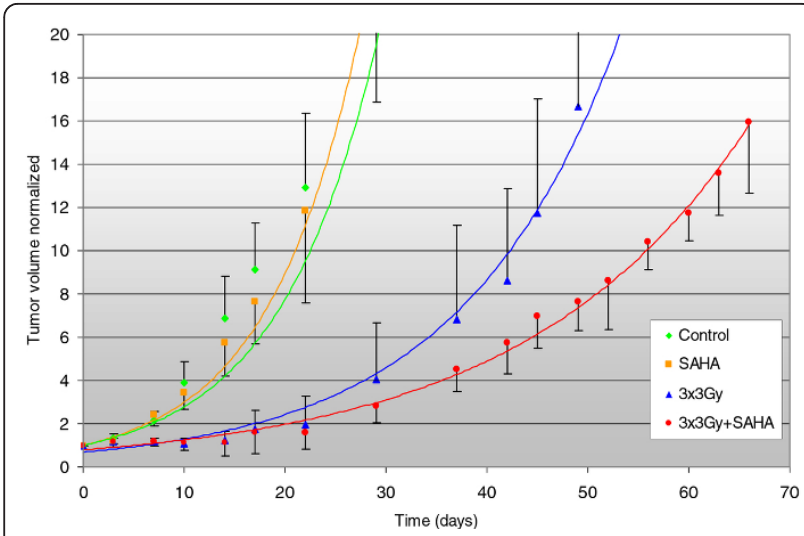

Figure 2 Tumor progression of malignant rhabdoid tumor xenografts (MRT) in Cohort II after treatment with ionizing radiation (XRT) or SAHA and XRT. The y-axis plots the

multiplication of initial tumor size normalized to the initial size $=1$, $x$-axis plots the time after treatment initiation in days. Figure 1 represents Cohort I and Figure 2 Cohort II. SAHA 100 mg/kg was injected intraperitoneally once daily for 8 consecutive days in Cohort I and for 15 days within 3 weeks in Cohort II starting with day 0. XRT with a single dose of $10 \mathrm{~Gy}$ (Cohort I) or with $3 \times 3$ Gy on three consecutive days (Cohort II) was delivered day $1(-3)$. After a follow-up of $>40$ days, there was a trend towards slower progression in the combined treatment group compared to xenotransplants treated with XRT alone in Cohort I $(p=0.3)$, and a significant difference in Cohort II. $(p<0.05)$.

experimental setting, neither after being given for 8 days (Cohort I) nor for 3 weeks (Cohort II) (Figure 4).

Actual tumor growth delay was calculated with $\left(\mathrm{T}_{\mathrm{x}^{-}}{ }^{-}\right.$ $\left.\mathrm{T}_{\mathrm{x}}\right) / \mathrm{T}_{\mathrm{x}}$ as the time taken for the irradiatied tumors ( $\mathrm{T}$ ') and the control tumors $(\mathrm{T})$ to $\mathrm{x}$-fold multiply their

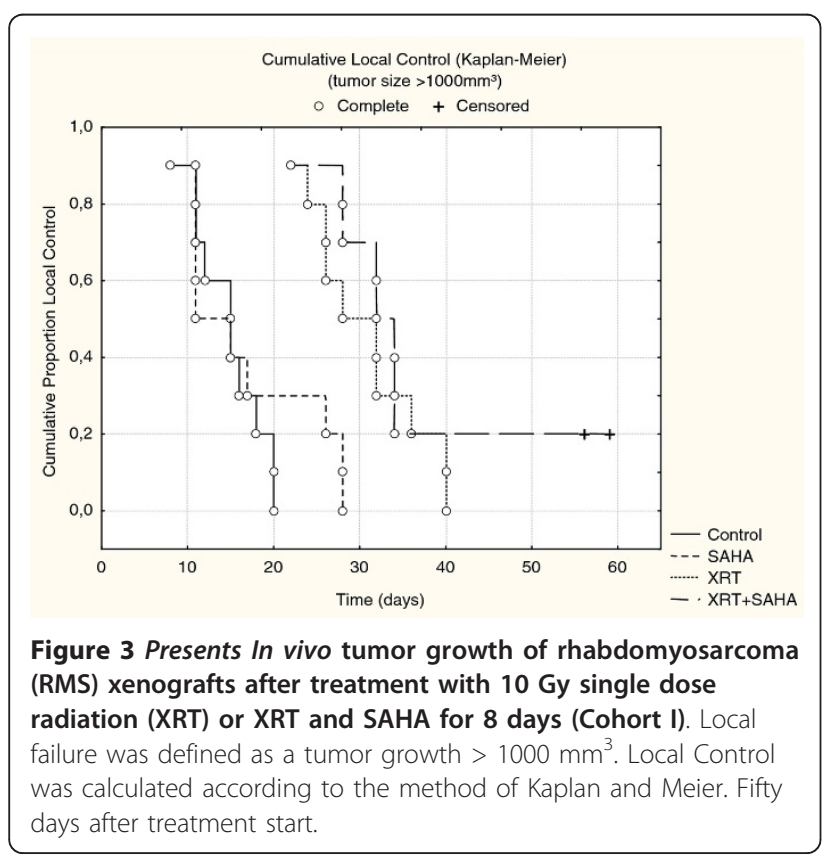

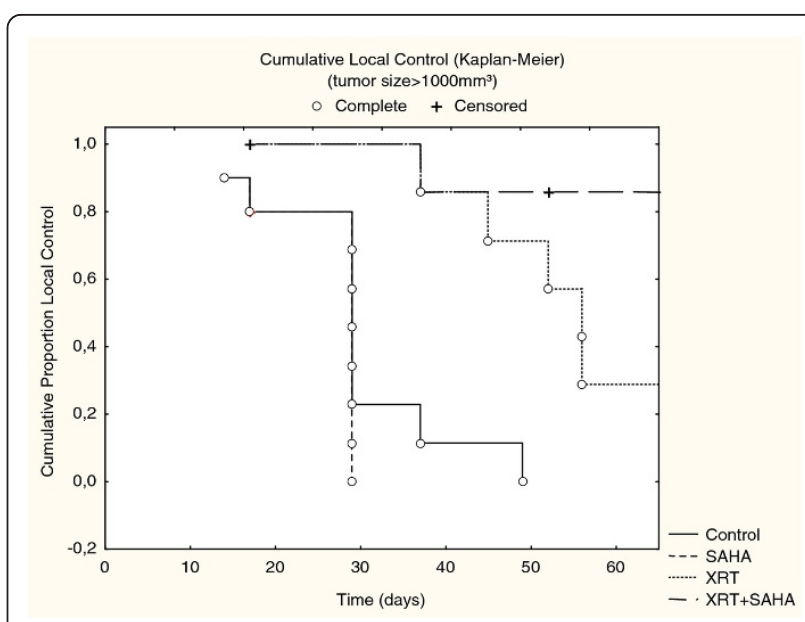

Figure 4 Presents In vivo tumor growth of rhabdomyosarcoma (RMS) xenografts after treatment with $3 \times 3 \mathrm{~Gy}$ fractionated radiation or XRT and SAHA for 3 weeks (Cohort II). Local failure was defined as a tumor growth $>1000 \mathrm{~mm}^{3}$. Local Control was calculated according to the method of Kaplan and Meier. Fifty days after treatment start.

volume $(\mathrm{x})$. Tumor growth delay was significant in Cohort II $(p<0.05)$, in which the parameters initial tumor size, fractionation of radio-treatment and period of SAHA application had been changed, but not in our initial Cohort I (Figure 5).

At the last day of treatment (day 8 and day 21 respectively), necrosis was observed in $25 \%$ of control tumors in Cohort I and $8 \%$ of tumors in Cohort II. This difference was attributed to the difference in initial tumor size at the time treatment was started. Interestingly in both cohorts any treatment had no statistically significant impact on the induction of necrosis. (Table 1, Figure 6). The analysis of Ki-67 expression on day 8 as well as day 21 showed significantly higher proliferation rates $(p<0.03, p<0.02)$ in the control tumors and the groups treated with SAHA alone compared to the groups treated with XRT \pm SAHA. However, no statistically significant difference between the XRT and XRT + SAHA group was observed in either cohort (Table 1, Figure 6).

Further, we investigated apoptosis on day 8 (cohort I) and day 21 (cohort II) in three tumors per treatment group by TUNEL test. Our results showed a significant increase of TUNEL positive cells in the tumors with combination treatment compared to tumors treated with XRT only ( $p=0.03$ (cohort I) and $p<0.0016$ (cohort II)) (Table 1).

We further evaluated induction of DSBs in the different treatment groups. It was previously demonstrated that DSB result in phosphorylation of H2AX on the $\gamma-$ site of serine 139 to form $\gamma \mathrm{H} 2 \mathrm{AX}$ [12-14]. We quantified the $\gamma \mathrm{H} 2 \mathrm{AX}$ expression in the xenograft tumors of three 

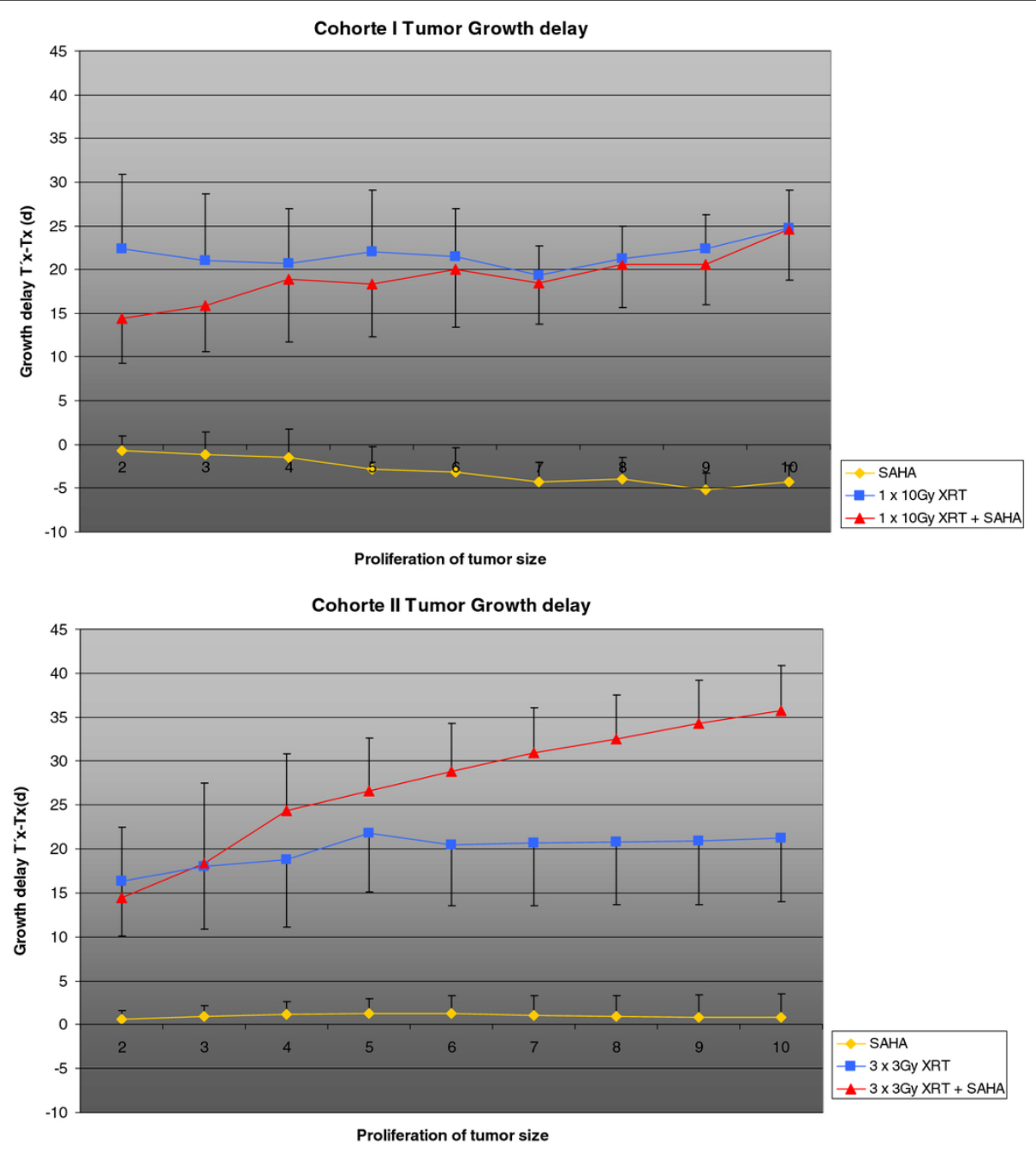

Figure 5 Actual tumor growth delay was calculated with $\left(T^{\prime}{ }^{\prime}-T_{x}\right) / T_{x}$ as the time taken for the irradiatied tumors $\left(T^{\prime}\right)$ and the control tumors (T) to $\mathbf{x}$-fold multiply their volume $(\mathbf{x})$. The $y$-Axis shows tumor growth delay in days, the $x$-axis shows the tumor growth $\left(T^{\prime}{ }^{\prime}-T_{x}\right) / T_{x}$. Tumor growth delay was significant in Cohort II $(p<0.05)$, but not in our initial Cohort I.

tumors per treatment group. The mice were treated with either vehicle control, SAHA $100 \mathrm{mg} / \mathrm{kg}$ for two consecutive days, XRT (10 Gy) or SAHA (100 mg/kg) on two consecutive days with XRT (10 Gy) on the second day of SAHA treatment. The mice were euthanized $30 \mathrm{~min}, 6 \mathrm{~h}, 24 \mathrm{~h}$ and $36 \mathrm{~h}$ after treatment, tumors were excised and analyzed for $\gamma \mathrm{H} 2 \mathrm{AX}$ expression using flow cytometry. After $30 \mathrm{~min}$, a single XRT dosage of $10 \mathrm{~Gy}$ resulted in a $3.4( \pm 1)$-fold induction of $\gamma \mathrm{H} 2 \mathrm{AX}$ expression compared to the vehicle control. Tumors of mice treated with XRT and SAHA showed a significantly higher induction $(p=0.03)$ of $\gamma \mathrm{H} 2 \mathrm{AX}$ expression $(8 \pm$

Table 1 Ki67-expression/Necrosis (\%) and Apoptosis (TUNEL test) in RMS xenografts treated with vehicle (DMSO), suberoylanilide hydroxamic acid (SAHA), irradiation (XRT), or SAHA and XRT in the first and second mouse cohort observed day 8 (cohort I) and day 21 (Cohort II)

\begin{tabular}{|c|c|c|c|c|c|c|c|c|}
\hline \multirow{3}{*}{$\begin{array}{l}\text { Treatment } \\
\text { Control }\end{array}$} & \multirow{2}{*}{\multicolumn{2}{|c|}{$\begin{array}{l}\text { Mean Tumor Age (days) } \\
\text { Cohort I Cohort II }\end{array}$}} & \multirow{2}{*}{\multicolumn{2}{|c|}{$\begin{array}{l}\text { Mean (SD) Ki-67 (\%) } \\
\text { Cohort I Cohortll }\end{array}$}} & \multirow{2}{*}{\multicolumn{2}{|c|}{$\begin{array}{l}\text { HE (necrosis)(\%) } \\
\text { Cohort I Cohortll }\end{array}$}} & \multirow{2}{*}{\multicolumn{2}{|c|}{$\begin{array}{l}\text { TUNEL (apoptosis) } \\
\text { Cohort I Cohortll }\end{array}$}} \\
\hline & & & & & & & & \\
\hline & $39( \pm 3)$ & 37 & $42( \pm 16)$ & $45( \pm 12)$ & $25( \pm 11)$ & $8( \pm 6)$ & $64( \pm 9)$ & $55( \pm 9)$ \\
\hline SAHA & $36( \pm 1)$ & 37 & $53( \pm 10)$ & $32( \pm 11)$ & $23( \pm 9)$ & $10( \pm 6)$ & $118( \pm 15)$ & $97( \pm 12)$ \\
\hline$X R T$ & $36( \pm 1)$ & 37 & $21( \pm 4)$ & $24( \pm 7)$ & $42( \pm 12)$ & $10( \pm 5)$ & $148( \pm 24)$ & $126( \pm 12)$ \\
\hline $\mathrm{SAHA}+\mathrm{XRT}$ & $36( \pm 1)$ & 37 & $14( \pm 8)$ & $17( \pm 4)$ & $48( \pm 17)$ & $12( \pm 6)$ & $201( \pm 11)$ & $192( \pm 10)$ \\
\hline
\end{tabular}




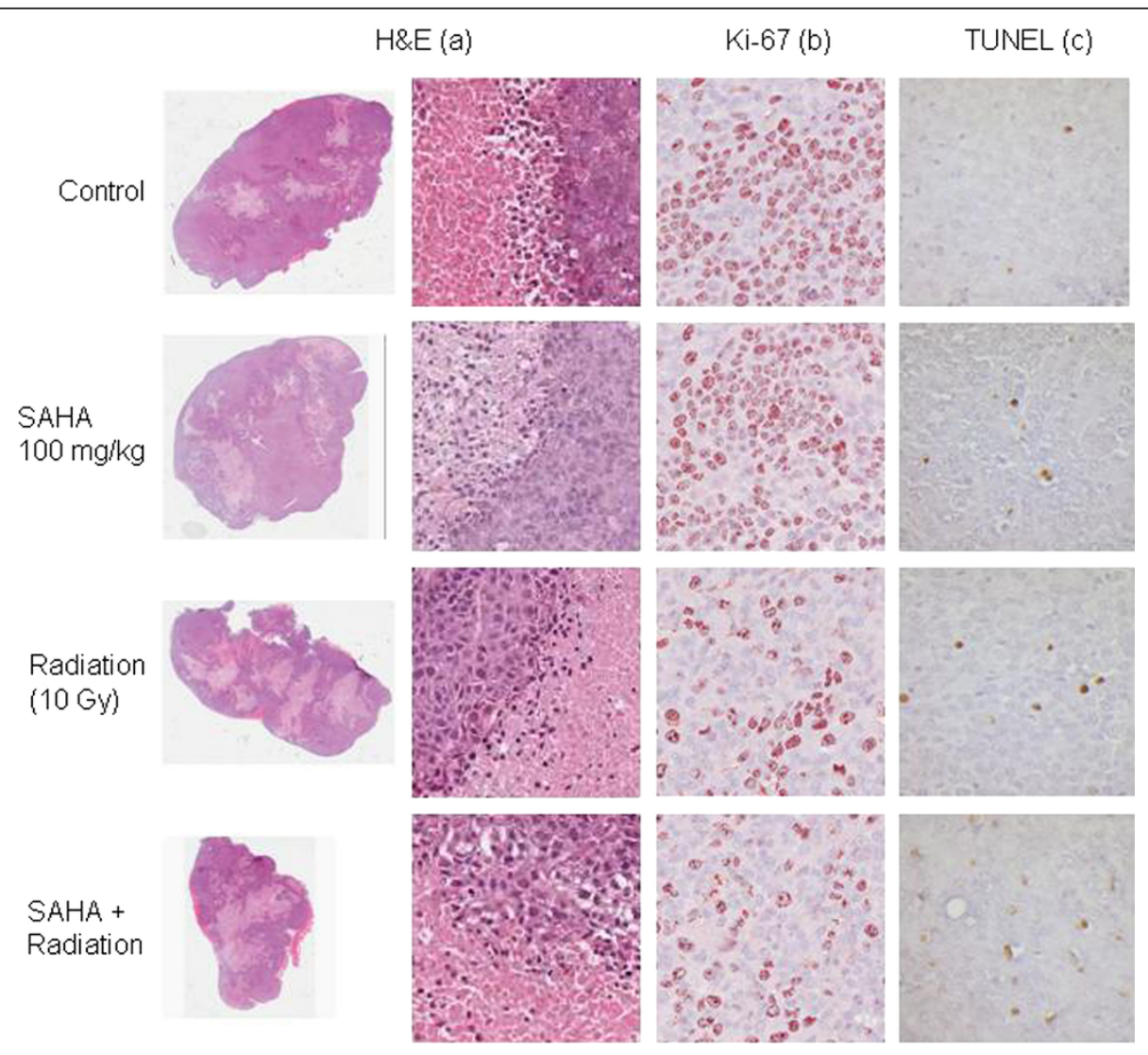

Figure $6 \mathrm{H} \& \mathrm{E}$, TUNEL and Ki67 staining of tumor tissue from MRT xenografts treated with vehicle, suberoylanilide hydroxamic acid (SAHA), ionizing radiation (XRT) or SAHA and XRT. See also Table 1.

1.5 -fold) compared to the vehicle control. SAHA given as a single agent resulted in no increase of $\gamma \mathrm{H} 2 \mathrm{AX}$ expression compared to the vehicle control (Figure 7). Investigation of further time points revealed that $\gamma \mathrm{H} 2 \mathrm{AX}$ expression decreased successively from $2 \mathrm{~h}$ to $6 \mathrm{~h}$ and $24 \mathrm{~h}$ after XRT. Interestingly $24 \mathrm{~h}$ after XRT, $\gamma \mathrm{H} 2 \mathrm{AX}$ expression of irradiated tumors reached the level of the untreated controls whereas the tumors of the combination group still showed an elevated expression level (2fold \pm 0.5 ).

Dynamic PET was used for tumor imaging to evaluate therapy response as previously described in animals after transfer of antiangiogenic genes [15]. In a preceding test four PET-tracers were tested in 6 mice with our sarcoma xenograft with every tracer being tested in 3 mice: FDG $\left({ }^{18} \mathrm{~F}\right.$-fluordeoxyglucose), FLT $\left({ }^{18} \mathrm{~F}\right.$-3'-deoxy3'-L-thymidine), ${ }^{68}$ Ga-RGD ( $\alpha v \beta 3 / \alpha v \beta 5$ integrin-binding peptide) and FET $\left({ }^{18} \mathrm{~F}\right.$-fluoro-ethyl-tyrosine). ${ }^{18} \mathrm{~F}$ FDG-PET showed the best results. Therefore, dynamic ${ }^{18} \mathrm{~F}$-FDG-PET was performed in 5 mice per treatment group before and on day 8 after treatment with SAHA, XRT or XRT plus SAHA (Table 2).
Comparing the results prior to and after treatment in each group there was no statistically significant effect on the standard uptake value (SUV) or on the transport of glucose into the tumor $(\mathrm{k} 1)$ or out of the tumor $(\mathrm{k} 2)$ in the different treatment groups. There was also no statistically significant impact on the hexokinase activity $(\mathrm{k} 3)$ by any treatment modality examined. The vascular fraction (VB) increased in the control group, but equally decreased in all treated tumor groups. Therefore, FDGPET did not seem to be an interesting option to monitor MRT treatment response in our mouse trial and was therefore discontinued after treatment of Cohort I.

\section{Discussion}

This is the first in vivo study that shows, that vorinostat has the potential to sensitize MRT to radiation.

Patients with malignant rhabdoid tumors still have a poor prognosis despite intensive current treatment protocols. Radiotherapy plays a significant role in local treatment, but is often delayed as long as possible in young children as late sequelae are feared. Therfore there is a need for new treatment strategies. 


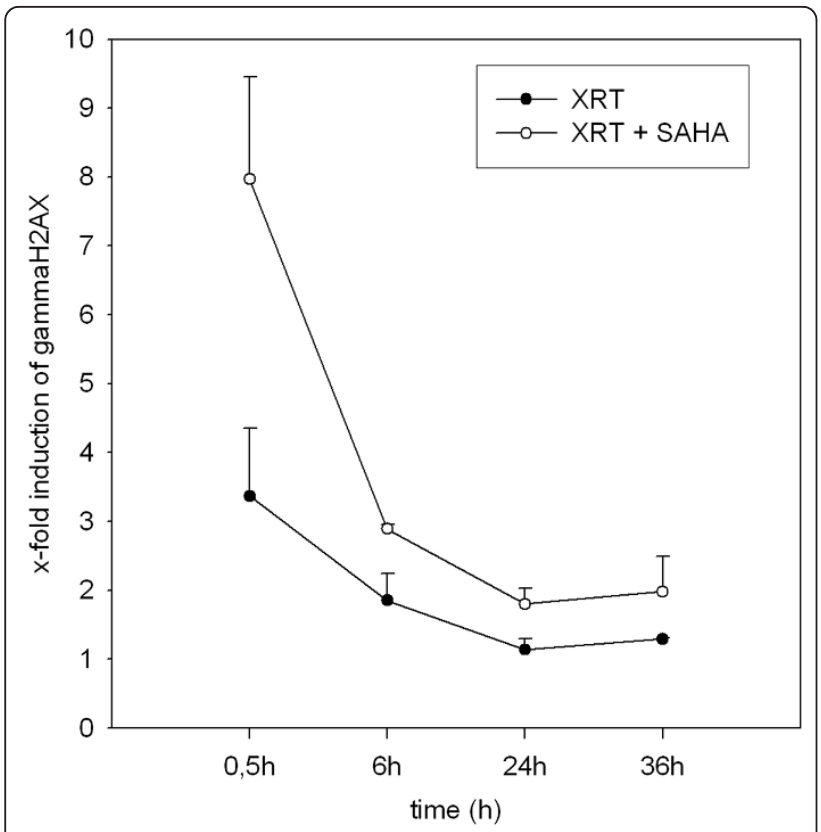

Figure $7 \gamma \mathrm{H} 2 \mathrm{AX}$ expression in MRT xenografts treated with ionizing radiation (XRT) or suberoylanilide hydroxamic acid (SAHA) and XRT. The mice were treated with either vehicle control, SAHA $100 \mathrm{mg} / \mathrm{kg}$ for two consecutive days, XRT (10 Gy) or SAHA $(100 \mathrm{mg} / \mathrm{kg})$ on two consecutive days with XRT (10 Gy) on the second day of SAHA treatment. After 30 min, tumors of mice treated with XRT and SAHA showed a significantly higher induction of $\gamma \mathrm{H} 2 \mathrm{AX}$ expression compared to tumors treated with XRT alone. $\gamma \mathrm{H} 2 \mathrm{AX}$ expression decreased successively from $2 \mathrm{~h}$ to $6 \mathrm{~h}$ and $24 \mathrm{~h}$ after XRT. $24 \mathrm{~h}$ after XRT, $\gamma \mathrm{H} 2 \mathrm{AX}$ expression of irradiated tumors reached the level of the untreated controls whereas the tumors of the combination group showed still an elevated expression level.

Epigenetic targeting therapies with HDACi have shown promising results in different tumor types. SAHA is one of the well-established HDACi which has been approved in the United States by the Food and Drug Administration (FDA) for the therapy for human cutaneous T-cell lymphoma. The drug can be given orally and side-effects are minor compared to cytotoxic chemotherapeutics. Therefore, it is an interesting candidate for tumor therapy. The promise of HDACi for cancer treatment, as a single agent and in combination with standard therapies, is supported by in vitro results of colorectal carcinoma, human melanoma and glioma cell experiments $[4,5,7]$. In several trials combination of HDACi with chemotherapy or radiotherapy improved tumor cell kill $[4,7,16,17]$. However, in vivo studies with HDACi as single-agent in cancer treatment, showed only moderate and limited efficacy [18].

Based on our promising in vitro results that showed a selectively radiosensitizing effect in the A-204 cell line used in this experiment as well as in two osteosarcoma cell lines, we now investigated the efficacy of the HDACi SAHA in combination with radiotherapy, compared to SAHA or XRT only in a MRT xenograft mouse model.

The results of our first Cohort did show a trend towards an improved local control and tumor growth delay in mice treated with SAHA plus XRT compared to animals treated with XRT alone. We attributed the lack of significance in Cohort I to three possible parameters: 1. short period of SAHA application, 2. fractionation of radiation and 3 . rather big tumor size at time of treatment initiation.

SAHA has previously been shown to augment the effects of radiotherapy in vivo tumor models $[3,6,16]$. In some of these in vivo studies, the drug was applied for several weeks with successful radiosensitization $[7,19]$, even though in others short-term application for 5 days and even single-dose treatment prior to irradiation seemed to be sufficient $[7,19]$.

The underlying pathways of HDACi acting as a radiosensitizer are still not completely understood [20]. Abrogation of DSB repair has been suggested to be one possible mechanism by several authors $[10,16]$. In our experiments, $\gamma \mathrm{H} 2 \mathrm{AX}$ expression, representing DNAdamage, was significantly influenced by the combination treatment. Interestingly, $\gamma \mathrm{H} 2 \mathrm{AX}$ expression of irradiated tumors reached the level of the untreated controls $24 \mathrm{~h}$ after XRT, whereas the tumors of the combination

Table 2 Descriptive statistics of the mean value and its standard deviation as well as the median values prior and after treatment of rhabdoid tumor xenografts with vehicle (DMSO), suberoylanilide hydroxamic acid (SAHA), irradiation (XRT) or XRT and SAHA

\begin{tabular}{|c|c|c|c|c|c|c|c|c|}
\hline \multirow[t]{2}{*}{ Parameter } & \multicolumn{2}{|l|}{ Control } & \multicolumn{2}{|l|}{ SAHA } & \multicolumn{2}{|l|}{ XRT } & \multicolumn{2}{|l|}{$\mathrm{SAHA}+\mathrm{XRT}$} \\
\hline & $\begin{array}{l}\text { Mean (SD) } \\
\text { prior }\end{array}$ & $\begin{array}{l}\text { Mean (SD) } \\
\text { after }\end{array}$ & $\begin{array}{l}\text { Mean (SD) } \\
\text { prior }\end{array}$ & $\begin{array}{l}\text { Mean (SD) } \\
\text { after }\end{array}$ & $\begin{array}{l}\text { Mean (SD) } \\
\text { prior }\end{array}$ & $\begin{array}{l}\text { Mean (SD) } \\
\text { after }\end{array}$ & $\begin{array}{l}\text { Mean (SD) } \\
\text { prior }\end{array}$ & $\begin{array}{l}\text { Mean (SD) } \\
\text { after }\end{array}$ \\
\hline$\overline{k 1}$ & $0.015(0.04)$ & $0.13(0.06)$ & $0.16(0.04)$ & $0.1(0.01)$ & $0.14(0.01)$ & $0.16(0.02)$ & $0.14(0.01)$ & $0.15(0.05)$ \\
\hline k2 & $0.32(0.07)$ & $0.38(0.02)$ & $0.37(0.12)$ & $0.24(0.08)$ & $0.33(0.03)$ & $0.34(0.02)$ & $0.39(0.03)$ & $0.39(0.02)$ \\
\hline k3 & $0.08(0.03)$ & $0.11(0.06)$ & $0.07(0.04)$ & $0.1(0.08)$ & $0.06(0.02)$ & $0.08(0.02)$ & $0.08(0.01)$ & $0.09(0.00)$ \\
\hline VB & $0.01(0.001)$ & $0.02(0.002)$ & $0.03(0.002)$ & $0.01(0.001)$ & $0.04(0.001)$ & $0.01(0.001)$ & $0.04(0.003)$ & $0.01(0.001)$ \\
\hline $\begin{array}{l}\text { Influx constant } \mathrm{Ki}(= \\
(\mathrm{k} 1 \times \mathrm{k} 3) /(\mathrm{k} 2+\mathrm{k} 3)\end{array}$ & 0.03 & 0.03 & 0.02 & 0.03 & 0.02 & 0.03 & 0.02 & 0.03 \\
\hline Max mSUV & 1323 (368) & $1593(631)$ & 2341 (1135) & 729 (305) & $1676(782)$ & 1578 (1206) & $1314(767)$ & $1158(816)$ \\
\hline
\end{tabular}


group showed still an elevated expression level (2-fold \pm $0.5)$, thus showing an impairment of repair kinetics by the combination treatment. Therefore, we assumed improved results after fractionated XRT and decided to treat Cohort II with $3 \times 3$ Gy instead of $1 \times 10$ Gy.

We wanted to use our first Cohort to investigate the potential of PET-imaging in our malignant rhabdoid tumor model. As the results were disappointing, we refrained from PET-imaging and thus were able to start treatment in smaller tumors in Cohort II.

These changes did indeed lead to a higher clinical impact resulting in significancy in Cohort II. As we decided to change all three parameters we can only guess which change has the highest impact and it remains unclear, if fractionation is obligatory or not. In previous reports on xenograft studies concerning breast tumors, neuroblastoma and ovarian cancer combination of HDACIs with fractionated $[7,17]$ as well as singledose irradiation [19] have been reported to be successful.

Interestingly - in contrast to reports concerning in vivo models of other tumor types $[16,17,20]$ - application of SAHA as a single agent had no effect in both of our experimental designs in this MRT xenotransplant model - neither when given for 8 days, nor for 3 weeks. Our SAHA dose, especially in Cohort II with $100 \mathrm{mg} / \mathrm{kg}$ for 5 consecutive days for 3 weeks is rather high compared to other studies $[7,17,19,20]$, in which SAHA proved to be successful. In these studies application differed from once $50 \mathrm{mg} / \mathrm{kg}, 3 \times 150 \mathrm{mg}$ within 1 week, $5 \times 12,5$ $\mathrm{mg} /$ week for 3 weeks and indeed $24 \times 50 \mathrm{mg}$ within 8 weeks. We therefore conclude from our study, that SAHA only treatment in MRT is not too promising.

We observed a strong trend towards a lower proliferation activity in the combination groups compared to the XRT alone groups. However, this trend failed to reach statistical significance in both cohorts.

Looking at necrosis of tumors, results showed no significant differences between the control group, SAHA group and the XRT or XRT plus SAHA group. However, apoptosis was indeed significantly higher in the $\mathrm{XRT}(p=0,001)$ as well as XRT + SAHA groups $(p=$ $0,0001)$ compared to the controls in both cohorts and also significantly higher in both XRT + SAHA groups compared to SAHA only $(p>0,0016)$. Thus, induction of apoptosis rather than necrosis seems to have the higher impact on radiosensitization by SAHA. However, as far as apoptosis was concerned the effect of SAHA + XRT seemed to be rather additive, while tumor growth delay in the mice seemed to be a rather synergistic effect given that SAHA alone had no significant effect at all. This proves again that apoptosis remains to be just one of the -all in all not completely understood - underlying mechanisms of radiosensitization.
We attribute the fact that no changes in our dynamic 18F-FDG-PET/CT images were to be observed to this lack of all investigated treatment schedules to induce significant necrosis in MRT. However, a further reason may be the initial tumor size (mean tumor size 300 $\mathrm{mm} 3$ ), which we chose in Cohort I in order to at all allow PET imaging [21], but which also may have attributed to the lower impact of the overall treatment that was observed in Cohort I compared to Cohort II.

\section{Conclusions}

SAHA is a promising radiosensitizer in malignant rhabdoid tumors, while it has no significant effect as a mono-agent. The addition of SAHA to radiotherapy does favorably and significantly influence apoptosis and DNA-repair kinetics in MRT xenotransplants, whereas necrosis is not influenced.

\section{Acknowledgements}

The skillfull technical assistance of Ludmilla Frick, Sylvia Trinh, Gabriele Becker, Alexandra Tietz, Angela Funk, Andreas Griesbach and Karin Leotta is gratefully acknowledged. The project was supported by the "Deutsche Krebshilfe" and the Dietmar Hopp Stiftung, Germany.

\section{Author details}

${ }^{1}$ Department of Radiation Oncology, University of Heidelberg, INF 600, 69120 Heidelberg, Germany. ${ }^{2}$ Institute of Pathology, University of Heidelberg, 69120 Heidelberg, Germany. ${ }^{3}$ Department of Radiation Oncology, German Cancer Research Center, 69120 Heidelberg, Germany. ${ }^{4}$ Department of Nuclear Medicine, University of Heidelberg and DKFZ, 69120 Heidelberg, Germany. ${ }^{5}$ Department of Pediatric Oncology, Hematology and Immunology, University Children's Hospital of Heidelberg, 69120 Heidelberg, Germany.

\section{Authors' contributions}

MT designed methods, carried out the experiments and analysed the data. SO co-designed the research theme, interpreted the results and wrote paper. VE helped to design of the flow-cytometry experiments and to interpret the results. WW and AS carried out the histopathological assessment in the tumor specimen and helped to interpret the results. MB and K-JW helped by discussing analyses, interpretation and presentation. RLP carried out and analyzed flow-cytometry assessment of $\mathrm{y}-\mathrm{H} 2 \mathrm{AX}$ experiments. $\mathrm{UH}$ designed and helped to analyze PET-experiments. AK co-designed the research theme. JD co-designed the research theme and provided the laboratory and radiation facilities. PEH co-analysed and co-discussed the data. CB designed the research theme, analysed and co-discussed all results. All authors contributed to and approved this manuscript.

\section{Competing interests}

The authors declare that they have no competing interests.

Received: 4 August 2011 Accepted: 29 March 2012

Published: 29 March 2012

\section{References}

1. Buscariollo DL, Park HS, Roberts KB, et al: Survival outcomes in atypical teratoid rhabdoid tumor for patients undergoing radiotherapy in a Surveillance Epidemiology, and End Results analysis. Cancer 2011.

2. Morgenstern DA, Gibson S, Brown T, et al: Clinical and pathological features of paediatric malignant rhabdoid tumours. Pediatr Blood Cancer 2010, 51(1):29-34.

3. Graham JS, Kaye SB, Brown R: The promises and pitfalls of epigenetic therapies in solid tumors. Eur J Cancer 2009, 45:1129-1136.

4. Folkvord S, Ree AH, Furre T, et al: Radiosensitization by SAHA in experimental colorectal carcinoma models - in viv effects and relevance 
of histone acetylation status. Int J Radiat Oncol Biol Phys 2009, 74(2):546-552.

5. Munshi A, Kurland JF, Nishikawa T, et al: Histone deacetylase inhibitors radiosensitize human melanoma by suppressing DNA repair activity. Clin Cancer Res 2005, 11:4912-4922.

6. Chinnaiyan $\mathrm{P}$, Vallabhaneni $\mathrm{G}$, Armstrong $\mathrm{E}$, et al: Modulation of radiation response by histone deacetylase inhibition. Int J Radiation Oncology Biol Phy 2005, 62:223-229.

7. Entin-Meer $M$, Yang $X$, Vandenburg SR, et al: In viv efficacy of a novel histone deacetylase inhibitor in combination with radiation for the treatment of gliomas. Neuro Oncol 2007, 9:82-88.

8. Blattmann C, Oertel S, Ehemann V, et al: Enhancement of radiation response in osteosarcoma and rhabdomyosarcoma cell lines by histone deacetylase inhibition. Int J Radiat Oncol Biol Phys 2010, 78:237-245.

9. Knipstein JA, Birks DK, Donson AM, et al: Histone deacetylase inhibition decreases proliferation and potentiates the effect of ionizing radiation in atypical teratoid/rhabdoid tumor cells. Neuro Oncol 2012.

10. Munshi A, Tanaka T, Hobbs ML, et al: Vorinostat, a histone deacetylase inhibitor, enhances the response of human tumor cells to ionizing radiation through prolongation of gamma-H2AX foci. Molecular Cancer Therapy 2006, 5:1967-1974.

11. Denecke T, Hundsdörfer P, Misch D, et al: Assessment of histological response of paediatric bone sarcomas using FDG PET in comparison to morphological volume measurement and standardized MRI parameters. Eur J Nucl Med Mol Imaging 2010, 37:1842-1853.

12. Paull TT, Rogakou EP, Yamazaki V, et al: A critical role for histone $H 2 A X$ in recruitment of repair factors to nuclear foci after DNA damage. Curr Biol 2000, 10:886-895.

13. Huang $X$, Darzynkiewicz Z: Cytometric assessment of histone H2AX phosphorylation: Method. Mol Biol 2006, 314:73-80.

14. Olive PL, Banath JP: Phosphorylation of histone H2AX as a measure of radiosensitivity. Int J Radiation Oncology Biol Phys 2003, 58:331-335.

15. Haberkorn U, Hoffend J, Schmidt $K$, et al: Changes in glucose metabolism and gene expression after transfer of anti-angiogenic genes in rat hepatoma. Eur J Nucl Med Mol Imaging 2007, 34(12):2011-2023.

16. Lopez G, Liu J, Ren W, et al: Combining PCI-24781, a novel histone deacetylase inhibitor, with chemotherapy for the treatment of soft tissue sarcoma. Clin Cancer Res 2009, 15(19):3472-3483.

17. Mueller S, Yang $X$, Sottero TL, et al: Cooperation of the HDAC inhibitor vorinostat and radiation in metastatic neuroblastoma: efficacy and underlying mechanisms. Cancer Lett 2011, 306(2):223-229.

18. Siu LL, Pili R, Duran I, et al: Phase I study of MGCD0103 given as a threetimes-per-week oral dose in patients with advanced solid tumors. J Clin Oncol 2008, 26:1940-1947.

19. Baschnagel A, Russo A, Burgan WE, et al: Vorinostat enhances the radiosensitivity of a breast cancer brain metastatic cell line grown in vitro and as intracranial xenografts. Mol Cancer Ther 2009, 8(6):1589-1595.

20. Chen MY, Liao WS, Lu Z, et al: Decitabine and suberoylanilide hydroxamic acid (SAHA) inhibit growth of ovarian cancer cell lines and xenografts while inducing expression of imprinted tumor suppressor genes, apoptosis, G2/M arrest, and autophagy. Cancer 2011, 16 DOl: 10.1002/ cncr.26073.

21. Arvanitis C, Bendapudi PK, Tseng JR, et al: (18)F and (18)FDG PET imaging of osteosarcoma to non-invasively monitor in situ changes in cellular proliferation and bone differentiation upon MYC inactivation. Cancer Biol Ther 2008, 7(12):1947-1951.

doi:10.1186/1748-717X-7-52

Cite this article as: Thiemann et al:: In vivo efficacy of the histone deacetylase inhibitor suberoylanilide hydroxamic acid in combination with radiotherapy in a malignant rhabdoid tumor mouse model. Radiation Oncology 2012 7:52.

\section{Submit your next manuscript to BioMed Central and take full advantage of:}

- Convenient online submission

- Thorough peer review

- No space constraints or color figure charges

- Immediate publication on acceptance

- Inclusion in PubMed, CAS, Scopus and Google Scholar

- Research which is freely available for redistribution

Submit your manuscript at www.biomedcentral.com/submit 